\title{
Social-affective features drive human representations of observed actions
}

\author{
Diana C. Dima ${ }^{1 *}$, Tyler Tomita ${ }^{2}$, Christopher Honey ${ }^{2}$, Leyla Isik ${ }^{1}$ \\ ${ }^{1}$ Dept. of Cognitive Science, Johns Hopkins University, United States \\ ${ }^{2}$ Dept. of Psychological and Brain Sciences, Johns Hopkins University, United States
}

\begin{abstract}
Humans observe actions performed by others in many different visual and social settings. What features do we extract and attend when we view such complex scenes, and how are they processed in the brain? To answer these questions, we curated two large-scale sets of naturalistic videos of everyday actions and estimated their perceived similarity in two behavioral experiments. We normed and quantified a large range of visual, action-related and socialaffective features across the stimulus sets. Using a cross-validated variance partitioning analysis, we found that social-affective features predicted similarity judgments better than, and independently of, visual and action features in both behavioral experiments. Next, we conducted an electroencephalography (EEG) experiment, which revealed a sustained correlation between neural responses to videos and their behavioral similarity. Visual, action, and social-affective features predicted neural patterns at early, intermediate and late stages respectively during this behaviorally relevant time window. Together, these findings show that social-affective features are important for perceiving naturalistic actions, and are extracted at the final stage of a temporal gradient in the brain.
\end{abstract}

\footnotetext{
* Correspondence should be addressed to: ddima@jhu.edu/diana.c.dima@gmail.com
} 


\section{Introduction}

2 In daily life, we rely on our ability to recognize a range of actions performed by others in a

3 variety of different contexts. Our perception of others' actions is both efficient and flexible,

4 enabling us to rapidly understand new actions no matter where they occur or who is performing

5 them. This understanding plays a part in complex social computations about the mental states

6 and intentions of others (Jamali et al., 2021; Spunt et al., 2011; Thornton et al., 2019; Thornton

7 and Tamir, 2021; Weaverdyck et al., 2021). Visual action recognition also interacts cross-

8 modally with language-based action understanding (Bedny and Caramazza, 2011; Humphreys et

9 al., 2013). However, there are two important gaps in our understanding of action perception in

10 realistic settings. First, we still don't know which features of the visual world underlie our

11 representations of observed actions. Second, we do not know how different types of action-

12 relevant features, ranging from visual to social, are processed in the brain, and especially how

13 they unfold over time. Answering these questions can shed light on the computational

14 mechanisms that support action perception. For example, are different semantic and social

15 features extracted in parallel or sequentially?

16 Relatively few studies have investigated the temporal dynamics of neural responses to actions.

17 During action observation, a distributed network of brain areas extracts action-related features

18 ranging from visual to abstract, with viewpoint-invariant responses emerging as early as $200 \mathrm{~ms}$

19 (Isik et al., 2018). Visual features include the spatial scale of an action (i.e. fine-scale

20 manipulations like knitting versus full-body movements like running) represented throughout

21 visual cortex (Tarhan and Konkle, 2020), and information about biological motion, thought to be

22 extracted within $200 \mathrm{~ms}$ in superior temporal cortex (Giese and Poggio, 2003; Hirai et al., 2003;

23 Hirai and Hiraki, 2006; Johansson, 1973; Jokisch et al., 2005; Vangeneugden et al., 2014).

24 Responses in occipito-temporal areas have been shown to reflect semantic features like invariant

25 action category (Hafri et al., 2017; Lingnau and Downing, 2015; Tucciarelli et al., 2019, 2015;

26 Wurm and Caramazza, 2019; Wurm and Lingnau, 2015), as well as social features like the

27 number of agents and sociality of actions (Tarhan and Konkle, 2020; Wurm et al., 2017; Wurm

28 and Caramazza, 2019). 
29 Among the visual, semantic, and social features thought to be processed during action

30 observation, it is unclear which underlie our everyday perception in naturalistic settings.

31 Mounting evidence suggests that naturalistic datasets are key to improving ecological validity

32 and reliability in visual and social neuroscience (Haxby et al., 2020; Nastase et al., 2020; Redcay

33 and Moraczewski, 2020). Most action recognition studies to date have used controlled images

34 and videos showing actions in simple contexts (Isik et al., 2018; Wurm and Caramazza, 2019).

35 However, presenting actions in natural contexts is critical, as stimulus-context interactions have

36 been shown to modulate neural activity (Willems and Peelen, 2021). Recent attempts to

37 understand naturalistic action perception, however, have yielded mixed results, particularly with

38 regard to the role of social features. For example, one recent study concluded that sociality (i.e.,

39 presence of a social interaction) was the primary organizing dimension of action representations

40 in the human brain (Tarhan and Konkle, 2020). Another, however, found that semantic action

41 category explained the most variance in fMRI data, with little contribution from social features

42 (Tucciarelli et al., 2019).

43 Here, we combined a new large-scale dataset of everyday actions with a priori feature labels to

44 comprehensively sample the hypothesis space defined by previous work. This is essential in light

45 of the conflicting results from previous studies, as it allowed us to disentangle the contributions

46 of distinct but correlated feature spaces. We used three-second videos of everyday actions from

47 the "Moments in Time" dataset (Monfort et al., 2019) and replicated our results across two

48 different stimulus sets. Action videos were sampled from different categories based on the

49 American Time Use Survey (ATUS, 2019) and were highly diverse, depicting a variety of

50 contexts and people. We quantified a wide range of visual, action-related, and social-affective

51 features in the videos and, through careful curation, ensured that they were minimally

52 confounded across our dataset.

53 We used this dataset to probe the behavioral and neural representational space of human action

54 perception. To understand the features that support natural action viewing, we predicted

55 behavioral similarity judgments using the visual, action-related, and social-affective feature sets.

56 Next, to investigate the neural dynamics of action perception, we recorded

57 electroencephalography (EEG) data while participants viewed the stimuli, and we used the three

58 sets of features to predict time-resolved neural patterns. 
59 We found that social-affective features predict action similarity judgments better than, and

60 independently of, visual and action-related features. Visual and action-related features explained

61 less variance in behavior, even though they included fundamental features such as the scene

62 setting and the semantic category of each action. Neural patterns revealed that behaviorally-

63 relevant features are automatically extracted by the brain in a progression from visual to action to

64 social features. Together, our results reveal the importance of social-affective features in how we

65 represent other people's actions, and show that these representations emerge in the brain along a

66 temporal gradient.

\section{Results}

\section{Disentangling visual, action, and social-affective features in natural videos}

69 We curated two sets of naturalistic three-second videos of everyday actions from the Moments in

70 Time dataset (Monfort et al., 2019). The videos were selected from a larger set, ensuring that

71 features of interest were minimally correlated (see Supplementary Methods). 18 common

72 activities based on the National Bureau of Labor Statistics' American Time Use Survey (ATUS,

73 2019) were represented (Table 1; see Methods, section Behavior: Stimuli). The two stimulus sets

74 contained 152 videos ( 8 videos per activity and 8 additional videos with no agents) and 65 videos

75 (3-4 videos per activity) respectively. The second set was used to replicate behavioral results in a

76 separate experiment with different stimuli and participants.

77 Naturalistic videos of actions can vary along numerous axes, including visual features (e.g. the

78 setting in which the action takes place), action-specific features (e.g. semantic action category),

79 and social-affective features (e.g. the number of agents involved or perceived arousal). To

80 evaluate these different axes, we quantified 17 visual, action-related and social-affective features

81 using image properties, labels assigned by experimenters, and behavioral ratings collected in

82 online experiments (Figure 1a). Visual features ranged from low-level (e.g. pixel values) to high-

83 level (e.g. activations from the final layer of a pretrained neural network). Action-related features

84 included transitivity (object-relatedness), activity (the amount of activity in a video), effectors

85 (body parts involved), and action category based on the American Time Use Survey (ATUS,

86 2019). Finally, social-affective features included sociality, valence, arousal and number of agents 
87 (see Methods, section Representational similarity analysis). Representational dissimilarity

88 matrices (RDM) were created for each feature by calculating pairwise Euclidean distances

89 between all videos.

90 In both video sets there were only weak correlations between visual features and the higher-level

91 action/social-affective features (Figure 1a). The highest correlations were those within each of

92 the three sets of features, including visual features (Exp 1: Conv1 and image saturation/gist,

$93 \tau_{A}=0.29 ;$ Exp 2: Conv1 and image hue, $\tau_{A}=0.32$ ), action features (Exp 1: arousal and activity,

$94 \tau_{A}=0.31$; Exp 2: activity and effectors, $\tau_{A}=0.33$ ) and social features (sociality and number of

95 agents; $\left.\operatorname{Exp} 1: \tau_{A}=0.31, \operatorname{Exp} 2: \tau_{A}=0.3\right)$.

96

a
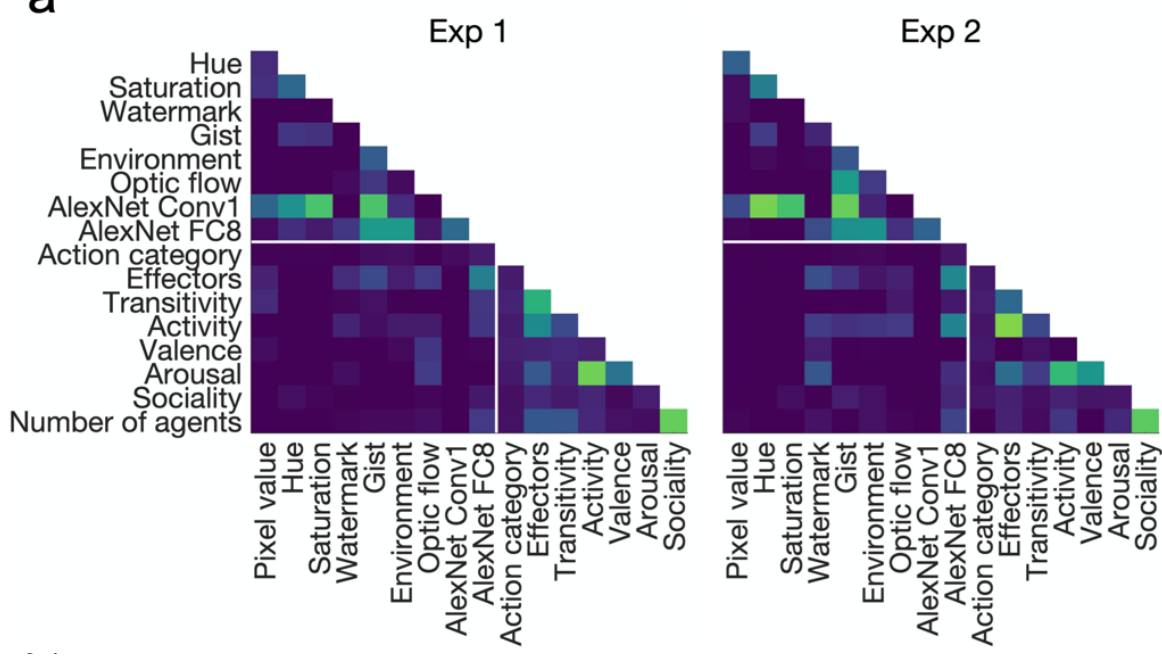

b

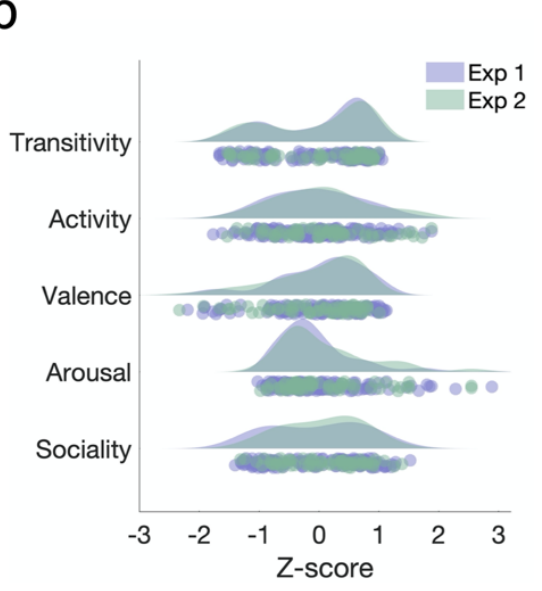

98 Figure 1. Quantifying visual, social-affective and action features in the two stimulus sets. a. Correlations between feature RDMs. Note the low correlations between visual features and action/social-affective features (white rectangle). b. Behavioral rating distributions in the two stimulus sets (plots: Allen et al., 2019).

101 The distributions of action and social-affective features were not significantly different between

102 the two stimulus sets (all Mann-Whitney $z<1.08, P>0.28$ ). The width of these distributions

103 suggests that the stimuli spanned a wide range along each feature (Figure 1b). In both

104 experiments, transitivity was notable through its bimodal distribution, likely reflecting the

105 presence or absence of objects in scenes, while other features had largely unimodal distributions. 


\section{Individual feature contributions to behavioral similarity}

108 To characterize human action representations, we collected behavioral similarity ratings for all

109 pairs of videos in each set in two multiple arrangement experiments (see Methods, section

110 Multiple arrangement). Participants arranged videos according to their similarity inside a circular

111 arena (Figure 2). The task involved arranging different subsets of 3-8 videos until sufficiently

112 reliable distance estimates were reached for all pairs of videos. Videos would play on hover, and

113 participants had to play and move each video to proceed to the next trial. In Experiment 1,

114 participants arranged different subsets of 30 videos out of the total 152, while in Experiment 2 ,

115 participants arranged all 65 videos. To emphasize natural behavior, participants were not given

116 specific criteria to use when judging similarity. Behavioral RDMs containing the Euclidean

117 distances between all pairs of stimuli were reconstructed from each participant's multiple

118 arrangement data using inverse MDS (Kriegeskorte and Mur, 2012).
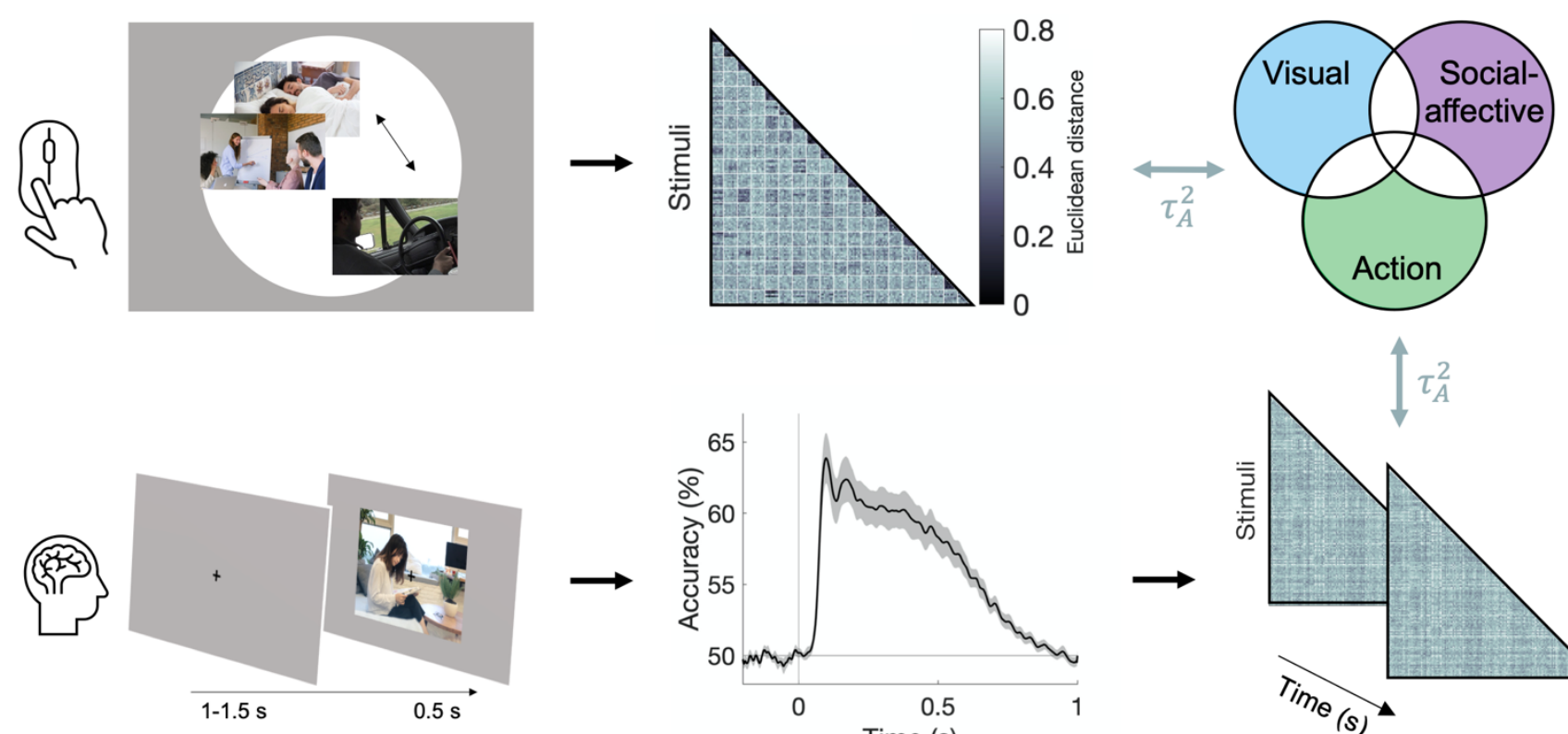

119
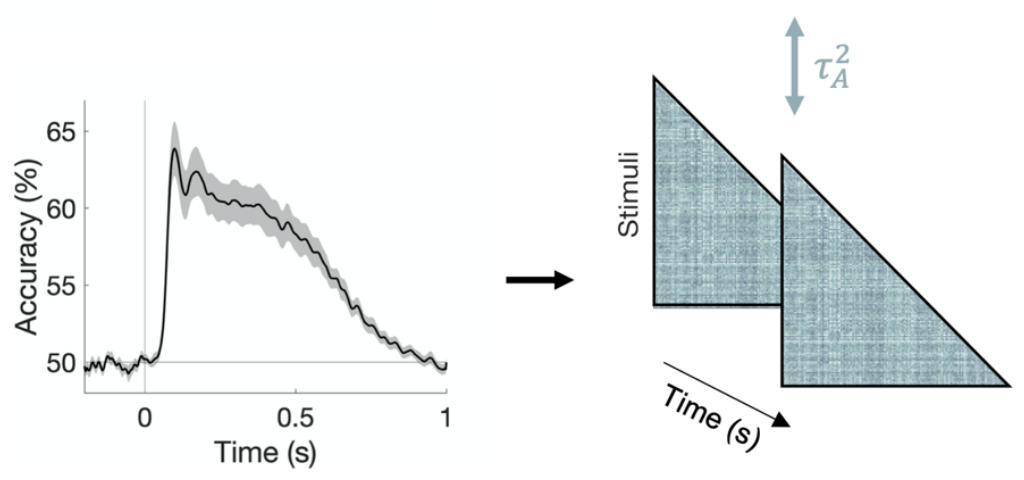

Figure 2. Experimental and analysis pipeline for evaluating the contribution of different features to action

121 representations. Above: a multiple arrangement task was used to generate behavioral RDMs in the two behavioral

122 experiments. Below: EEG data was recorded during a one-back task, and time-resolved neural RDMs were

123 generated using pairwise decoding accuracies. Cross-validated variance partitioning was used to assess the unique

124 contributions of visual, social-affective, and action features to the behavioral and neural RDMs, quantified as the

125 predicted squared Kendall's $\tau_{A}$. The stimuli in this figure are public domain images similar to the types of videos 
127 Data reliability was quantified using leave-one-subject-out correlations of the similarity ratings

128 and was above chance in both experiments (Kendall's $\tau_{A}=0.13 \pm 0.08$ and $0.18 \pm 0.08$

129 respectively, both $P<0.001$, permutation testing; Supplementary Figure 1a). Reliability was

130 significantly higher in Experiment 2 than in Experiment 1 (Mann-Whitney $z=3.21, P=0.0013$ ),

131 potentially reflecting differences in both participant pools and sampling methods (subsets of

132 videos in Experiment 1 versus full video dataset in Experiment 2; see Methods, section Multiple

133 arrangement).

134 We assessed the contribution of 17 different visual, social, and action features to behavior in

135 both experiments by correlating each feature RDM to each participant's behavioral RDM

136 (Supplementary Table 2). In Experiment 1 (Figure 3), only two visual features were significantly

137 correlated with the behavioral RDMs (environment and activations from the final fully-

138 connected layer FC8 of AlexNet). However, there were significant correlations between

139 behavioral RDMs and all action-related RDMs (action category, effectors, transitivity and

140 activity), as well as all social-affective RDMs (valence, arousal, sociality and number of agents).

141

142

143

144
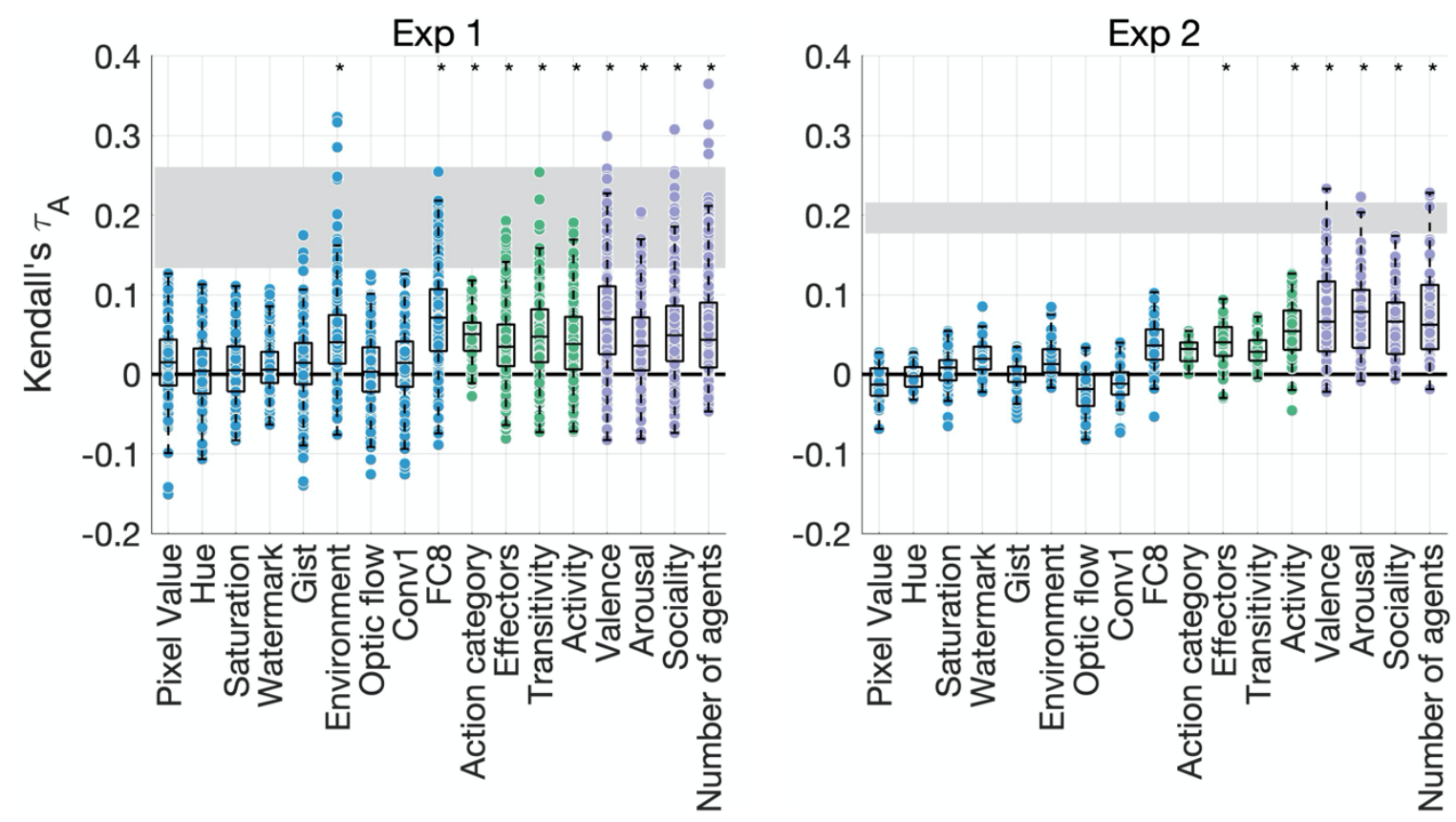

Figure 3. Feature contributions to behavioral similarity. The correlations between each feature RDM and the behavioral RDMs are plotted against the noise ceiling (gray). Each dot is an individual subject. Asterisks denote significance $(P<0.005)$. 
145 In Experiment 2, the only visual feature that contributed to behavior was the final fully-

146 connected layer of AlexNet (Figure 3). Among action features, only effectors and activity were

147 significantly correlated with the behavioral RDMs. However, we found significant correlations

148 with all social-affective features. The results thus converge across both experiments in

149 suggesting that social-affective and, to a lesser extent, action-related features, rather than visual

150 properties, explain behavioral similarity.

\section{Social-affective features explain the most unique variance in behavioral representations}

152 We performed a cross-validated variance partitioning analysis (Groen et al., 2018; Lescroart et

153 al., 2015; Tarhan et al., 2021) to determine which features contributed the most unique variance

154 to behavior (see Methods, section Variance partitioning). We selected the ten features that

155 contributed significantly to behavior in either experiment, i.e. two visual features (environment

156 and layer FC8 of AlexNet) and all action and social-affective features. To keep the analysis

157 tractable and understand the contribution of each type of information, we grouped these features

158 according to their type (visual, action and social-affective) and used them as predictors in a

159 cross-validated hierarchical regression (Figure 4). Note that there was no collinearity among the

160 ten predictors, with an average variance inflation factor of 1.34 (Experiment 1) and 1.37

161 (Experiment 2).

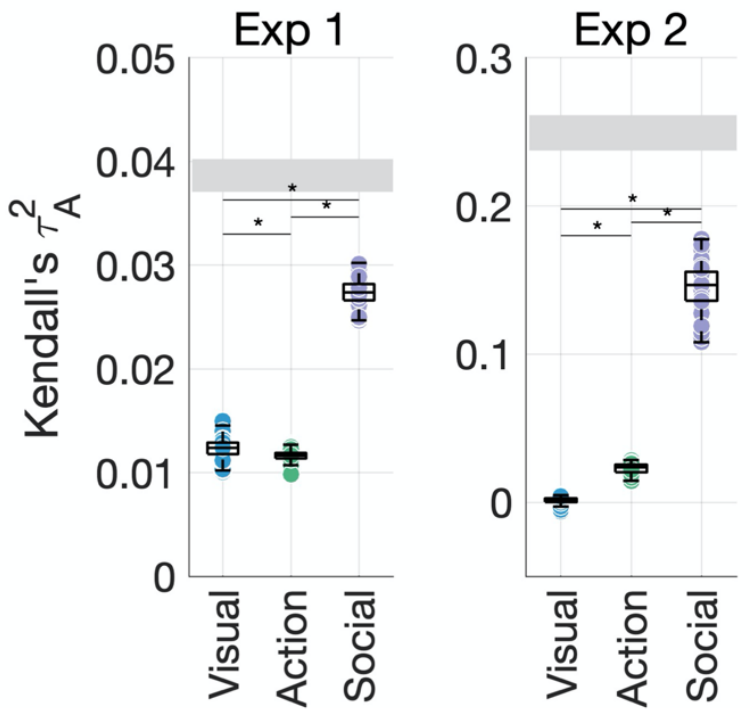


Figure 4. Social-affective features explain behavior better than visual and action features. The unique variance explained by visual, action, and social-affective features is plotted against the split-half reliability of the data (gray).

166 Together, the ten predictors explained most of the systematic variance in behavior. In

167 Experiment 1, the predicted squared Kendall's $\tau_{A}$ of the full model was higher on average than

168 the true split-half squared correlation $\left(\tau_{A}^{2}=0.06 \pm 0.001\right.$ and $\tau_{A}^{2}=0.04 \pm 0.002$ respectively). This is

169 likely to be due to the lower reliability of the behavioral similarity data in this experiment, and

170 suggests that the ten predictors are able to explain the data well despite the overall lower

171 prediction accuracy. In Experiment 2, the full model achieved a predicted $\tau_{A}^{2}$ of $0.18 \pm 0.1$ on

172 average, compared to a true squared correlation of $0.25 \pm 0.1$, suggesting that the ten predictors

173 explain most of the variance $(73.21 \%)$ in the behavioral data.

174 In both experiments, social-affective features contributed significantly more unique variance to

175 behavior than visual or action features (Figure 4, all Wilcoxon $z>5.5$, all $P<0.001$ ). While all

176 three groups of features contributed unique variance to behavior in Experiment 1 (all $P<0.001$,

177 randomization testing), in Experiment 2, only social-affective features contributed significantly

178 to behavior $(P<0.001)$, while visual and action features did not $(P=0.06$ and $P=0.47$

179 respectively). Shared variance between feature groups was not a significant contributor in either

180 dataset.

181 The semantic RDM included among the action features was a categorical model based on 182 activity categories (ATUS, 2019). To assess whether a more detailed semantic model would 183 explain more variance in behavior, we generated a feature RDM using WordNet similarities

184 between the verb labels corresponding to the videos in the Moments in Time dataset. However, 185 replacing the action category RDM with the WordNet RDM did not increase the variance 186 explained by action features (Supplementary Figure 2).

187 Among the social-affective features we tested, the number of agents could be seen as straddling 188 the visual and social domains. To assess whether our results were driven by this feature, we 189 performed a control variance partitioning analysis pitting the number of agents against the other,

190 higher-level social-affective features (Supplementary Figure 3). In both experiments, the higher- 
191 level features (sociality, valence and arousal) contributed more unique variance than the number

192 of agents, suggesting that our results are not explained by purely visual factors.

194 EEG patterns reflect behavioral similarity

195 We performed an EEG experiment to investigate how action-relevant features are processed over

196 time. Participants viewed $500 \mathrm{~ms}$ segments of the 152 videos from Experiment 1 and performed

197 a one-back action task in which they detected repetitions of the action category (see Methods,

198 section EEG: Experimental procedure). To relate neural patterns to behavioral and feature

199 RDMs, we computed time-resolved neural RDMs for each participant using decoding accuracies

200 between all pairs of videos (Figures 2, 5a). The time-course of decoding performance was similar

201 to that observed in previous E/MEG studies using still visual stimuli (Carlson et al., 2013; Cichy

202 et al., 2014; Dima et al., 2018; Greene and Hansen, 2018; Isik et al., 2014). Decoding accuracy

203 rose above chance at $50 \mathrm{~ms}$ after video onset, reached its maximum at $98 \mathrm{~ms}(63.88 \pm 6.82 \%$

204 accuracy), and remained above chance until $852 \mathrm{~ms}$ after video onset (cluster-corrected $P<0.05$, 205 sign permutation testing).
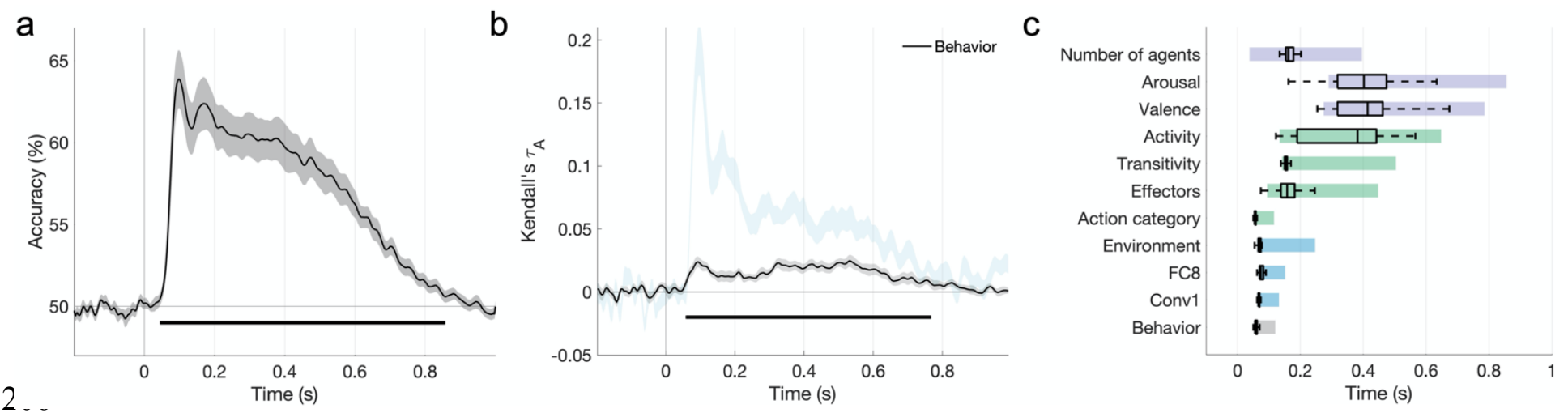

207 Figure 5. The features that explain behavioral action representations also contribute to neural representations. a.

208 Time-course of video decoding accuracy, averaged across all pairs of videos and participants (in gray: SEM across

209 participants). The horizontal line marks above-chance performance (sign permutation testing, cluster-corrected

$210 P<0.05)$. b. Behavioral similarity correlates with the neural RDM. The noise ceiling is shown in light blue (leave-

211 one-subject out correlation, mean $\pm \mathrm{SD})$. Horizontal lines mark significant time windows $(P<0.05$, cluster-corrected).

212 c. The distribution of significant correlation onsets for each feature model across 1000 bootstrapping iterations

$213(P<0.05$, cluster-corrected $)$. Color rectangles show $90 \%$ confidence intervals. 
214 To assess brain-behavior correlations, we related the average behavioral RDM obtained in

215 Experiment 1 to the time-resolved neural RDMs (Kendall's $\tau_{A}$ ). The behavioral RDM correlated

216 significantly with neural patterns during a cluster between 62 and $766 \mathrm{~ms}$ after video onset

217 (Figure 5b), suggesting that the features guiding the intuitive categorization of naturalistic

218 actions also underlie their neural organization.

\section{Neural timescale of individual feature representations}

220 We assessed the correlations between EEG patterns and the ten feature RDMs found to

221 contribute to behavior in Experiment 1. We also included an additional feature RDM based on

222 the first convolutional layer of AlexNet, which best captures early visual neural responses

223 (Supplementary Figure 7; see Methods, section Multivariate analysis). The feature RDMs that

224 contributed to behavioral similarity also correlated with the EEG patterns (Supplementary

225 Figures 7-9), with a single exception (sociality).

226 A bootstrapping analysis of the cluster onsets of these correlations (Figure 5c) suggests a

227 progression from visual to action and social-affective features. Visual predictors correlated with

228 the neural patterns between $65 \pm 15 \mathrm{~ms}$ (mean $\pm \mathrm{SD}$, Conv1) and 84 $\pm 62 \mathrm{~ms}$ (Environment),

229 while action category also had an early onset $(58 \pm 9 \mathrm{~ms})$. Other action-related features, however,

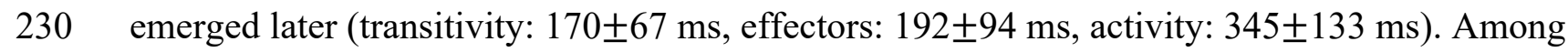

231 social-affective features, the number of agents had the earliest correlation onset $(178 \pm 81 \mathrm{~ms})$,

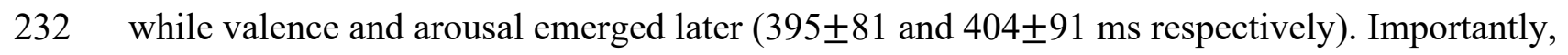

233 these features are spontaneously extracted in the brain, as none of them, with the exception of

234 action category, were directly probed in the one-back task performed by participants. In addition,

235 all features were extracted during behaviorally relevant time windows (Figure 5b).

\section{A temporal hierarchy in action perception}

237 A cross-validated variance partitioning analysis revealed different stages in the processing of

238 naturalistic actions (Figure 6). Visual features dominated the early time windows (66-138 ms

239 after video onset). Action features also contributed a significant amount of unique variance (162-

$240598 \mathrm{~ms}$ ), as well as variance shared with social-affective features (354-598 ms; Supplementary

241 Figure 5). Finally, social-affective features independently predicted late neural responses (446- 
$242782 \mathrm{~ms}$ ). Importantly, visual features did not share a significant amount of variance with either

243 action or social-affective features.

244 An analysis of effect onsets across 100 split-half iterations points to the hierarchical processing 245 of these features, with a progression from visual to action to social-affective features. Social-

246 affective features (mean onset $418 \pm 89 \mathrm{~ms}$ ) contributed unique variance significantly later than

247 other feature sets, while action features $(245 \pm 104 \mathrm{~ms})$ came online later than visual features

248 (65 $\pm 8 \mathrm{~ms}$; all Wilcoxon $z>7.27, P<0.001$; Figure $6 \mathrm{~b})$. A fixed-effects analysis revealed the same

249 order of feature information with larger effect sizes (Supplementary Figure 6).

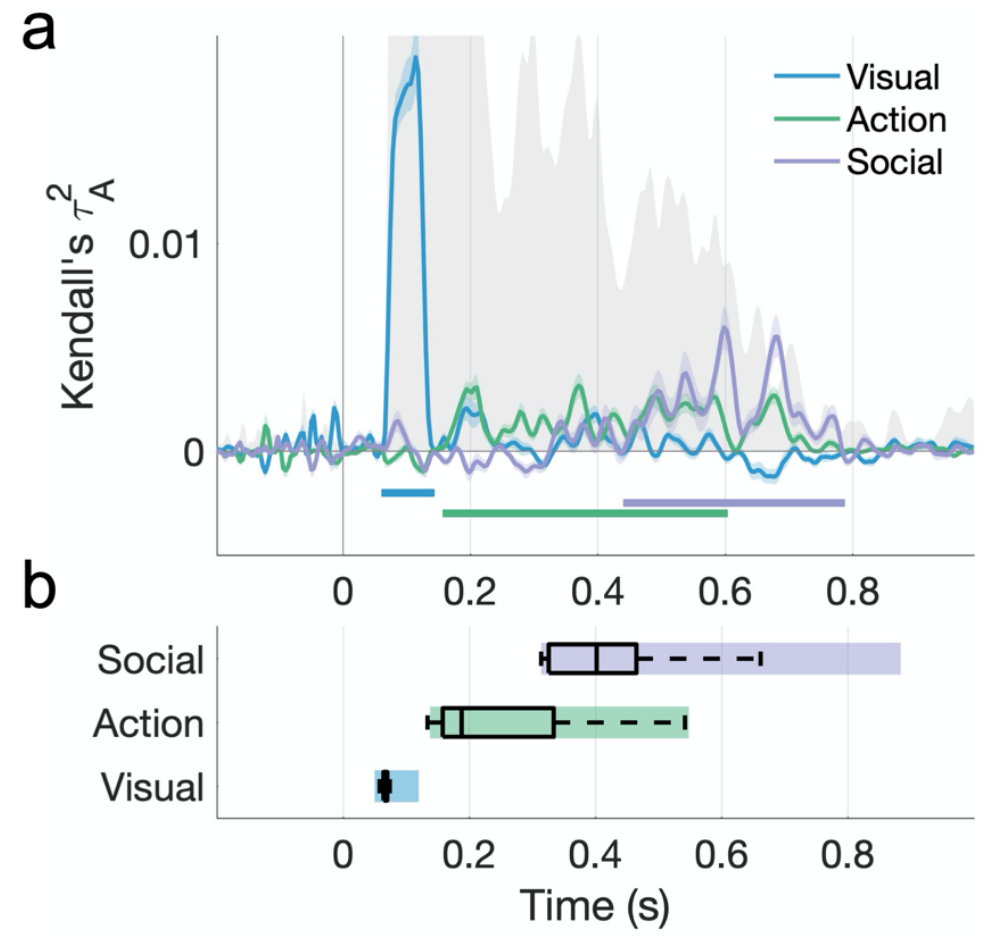

251 Figure 6. Hierarchical processing of visual, action, and social-affective features. a. Unique variance explained by each group of features over time. The split-half reliability of the data is shown in gray (shaded area; see also Figure

$5 b)$. b. The distribution of effect onsets across 100 split-half iterations. Color rectangles show $90 \%$ confidence intervals.

\section{Discussion}

256 Here, we used a large-scale naturalistic stimulus set to disentangle the roles of different features

257 in action perception. Two novel findings emerge from our study. First, our behavioral results 
suggest that social-affective features play the most important role in how we organize naturalistic everyday actions, above and beyond fundamental visual and action features like scene setting or action category. Second, these behaviorally-relevant features are spontaneously extracted in the brain, and follow a hierarchical sequence from visual to action-related and culminating with social-affective features. These results offer an account of how internal representations of everyday actions emerge in the mind and brain.

\section{Behavioral representations: what features support action perception?}

Across two separate multiple arrangement experiments with large-scale naturalistic stimulus sets,

266 we found that social-affective features predicted similarity judgments better than, and

267 independently of, visual and action-related features. By sampling a comprehensive feature space

268 ranging from low-level to conceptual, we were able to distinguish between components that

269 often covary, such as scene setting and action category or sociality and transitivity. Previous

270 studies have operationalized features in different ways, and an exhaustive investigation is thus

271 difficult; however, our approach of including several important features from each group

272 mitigated this, as suggested by the high amount of variance in behavior collectively explained by

273 our features.

274 Our work adds to a growing body of evidence for the importance of social features in action

275 perception, and extends it by disentangling the contributions of specific social and semantic

276 features. Previous work has highlighted sociality as an essential feature in neural action

277 representations (Tarhan and Konkle, 2020; Wurm et al., 2017; Wurm and Caramazza, 2019) and

278 a recent study (Tarhan et al., 2021) found that behavioral action similarity judgments were better

279 explained by similarity in actors' goals than by visual similarity. In line with this work, we found

280 a minimal contribution of visual features to action similarity judgments. In contrast, all of our

281 social-affective features - the number of agents, sociality, valence, and arousal - were

282 significantly correlated with behavioral similarity. Furthermore, only two individual action-

283 related features replicated across the two experiments: the amount of activity and the effector

284 (body part) feature, the latter of which is highly relevant to the actors' goals. This could be

285 interpreted as further evidence for the importance of socially-relevant features in our internal 
representations of actions, and identifies specific social and goal-related features that are important for action understanding.

A hypothesis-driven approach will always pose challenges, due to practical limitations in the number of feature spaces one can feasibly test. Our approach of grouping predictors together

290 based on theoretical distinctions made it possible to rigorously evaluate the unique contributions

291 of different types of features, which is an essential first step in understanding naturalistic action

292 representations. This analysis revealed that social-affective features contributed the most unique

293 variance in both experiments, suggesting that they robustly predict behavioral similarity

294 judgments, while visual and action features explained little unique variance in either experiment

295 (Figure 4).

296 Our social-affective feature space included one feature that could be construed as a perceptual 297 precursor to sociality, namely the number of agents in each video. Indeed, previous fMRI work

298 has suggested that neural representations of actions in the visual system reflect perceptual

299 precursors of social features, rather than higher-level social features (Wurm and Caramazza,

300 2019). Here, we found that high-level social-affective features (sociality, valence and arousal)

301 contributed significantly to behavior independently of the number of agents. Further, these high-

302 level social-affective features explained significantly more unique variance in behavior than the 303 number of agents in both experiments (Supplementary Figure 3). Our findings suggest that high-

304 level social-affective features uniquely drive human action representations.

\section{Neural representations: how does action perception unfold over time?}

306 Using EEG, we tracked the temporal dynamics of naturalistic action perception. Using

307 naturalistic stimuli and a rich feature space enabled us to disentangle the contributions of

308 different features and investigate their relative timing. Visual, action, and social-affective

309 features made unique contributions to the EEG patterns at different processing stages, revealing a

310 representational hierarchy of spontaneously-extracted features.

311 Almost all behaviorally-relevant features correlated with the EEG patterns, with action-related and

312 social-affective features emerging later than visual features (Figure 5c). Most action-related features

313 emerged within $200 \mathrm{~ms}$, on the timescale of feedforward processing, which is consistent with prior work 
314 showing invariant responses to actions as early as $200 \mathrm{~ms}$ (Isik et al., 2018; Tucciarelli et al., 2015), and 315 action transitivity processing as early as $250 \mathrm{~ms}$ (Wamain et al., 2014). Among social-affective features,

316 the number of agents emerged earliest $(162 \mathrm{~ms})$, pointing to the role of this feature as a perceptual

317 precursor in social perception (Papeo, 2020; Wurm and Caramazza, 2019). Valence and arousal emerged

318 later, around $400 \mathrm{~ms}$ after video onset. Interestingly, sociality, which has been highlighted as an

319 important dimension in previous fMRI work on action perception (Tarhan and Konkle, 2020; Wurm et

320 al., 2017), did not correlate with the EEG patterns. While the absence of an effect does not preclude the

321 possibility that this feature is being processed, it is possible that prior work has confounded sociality

322 with other correlated social-affective features (such as the number of agents, or arousal).

323 Variance partitioning revealed a clear temporal progression from visual features ( $\sim 100 \mathrm{~ms})$ to 324 action features ( 150-600 ms) to social-affective features ( 400-600 ms). Importantly, these

325 processing stages emerged after partialling out the contributions of other groups of predictors in

326 a cross-validated analysis, validating our a priori distinctions between feature classes. These

327 findings suggest that the extraction of visual features occurs rapidly, within $200 \mathrm{~ms}$, and is likely

328 supported by feedforward computations. The social-affective features that support behavioral

329 representations, however, were extracted last. This is consistent with theories suggesting that

330 internal visual experience reverses the stages of perceptual processing (Dijkstra et al., 2020;

331 Hochstein and Ahissar, 2002). Specifically, it was the final, social-affective stage of neural

332 processing that was reflected in the intuitive behavioral representations, and not the initially

333 extracted visual features. Furthermore, action-related features were extracted significantly before

334 social-affective features, suggesting the two are not extracted in parallel, but instead pointing to a

335 hierarchy in which both visual and action-related features may contribute to socially relevant

336 computations.

337 Our results add temporal characterization to previous fMRI findings, suggesting that the 338 seemingly conflicting features revealed by previous studies, like sociality (Tarhan and Konkle,

339 2020) or semantic action category (Tucciarelli et al., 2019), emerge at different stages during

340 action observation. Thus, the existence of different organizing dimensions can be explained not

341 just through spatial segregation within and across brain areas, but also through a temporal

342 gradient starting with visual features and concluding with behaviorally-relevant social

343 representations. More work is needed to understand where these dynamic representations emerge 
344 in the brain, and whether they are supported by overlapping or distinct networks. Future research

345 could test this using EEG-fMRI fusion to track the spatiotemporal dynamics of action

346 representations.

\section{Actions in context}

348 As real-world actions tend to occur in a rich social context, studies of action perception should

349 consider social features and the interactions between different systems for perceiving actions,

350 agents and their mental states (Quadflieg and Koldewyn, 2017). Recent works suggests that

351 social perception enhances visual processing (Bellot et al., 2021; Papeo, 2020) and recruits

352 dedicated neural circuits (Isik et al., 2017; Pitcher and Ungerleider, 2021). Our findings open

353 exciting new avenues for connecting these areas of research. For example, future studies could

354 more explicitly disentangle the perceptual and conceptual building blocks of social and affective

355 features, such as body posture or facial expression, and their roles in action and interaction

356 perception.

357 One fundamental question that lies at the root of this work is how actions should be defined and

358 studied. Here, we adopted a broad definition of the term, focusing on activities as described in

359 the American Use Survey (ATUS, 2019). Although our stimuli were selected to clearly depict

360 short, continuous actions performed by visible agents, their naturalistic and context-rich nature

361 means that they could be understood as "events", encompassing elements that are not singularly

362 specific to actions. A wealth of evidence has shown that context changes visual processing in a

363 non-additive way (Bar, 2004; Willems and Peelen, 2021), and emerging evidence suggests that

364 the same is true for actions (Wurm et al., 2012). Studying actions in context holds promise for

365 understanding how semantically rich representations emerge in naturalistic vision. This, in turn,

366 will pave the way towards a computational understanding of the neural processes that link

367 perception and cognition. 


\section{Methods}

Behavior: Stimuli

370 We curated two stimulus sets containing three-second videos of everyday actions from the

371 Moments in Time dataset (Monfort et al., 2019). To broadly sample the space of everyday

372 actions, we first identified the most common activities from the National Bureau of Labor

373 Statistics' American Time Use Survey (ATUS, 2019). We adjusted this list to include both social

374 and non-social activities that lend themselves to visual representation (Table 1). In particular,

375 "educational activities" were included both under "working" and a more specific "instructing"

376 category. In addition, some broad categories ("leisure and sports", "household activities") were

377 split into more specific ones. Finally, we added a "fighting" category to capture unpleasant social

378 interactions, and a "driving" category (as travel was only included under "other activities") .

\begin{tabular}{|l|l|}
\hline Activity & Verb labels \\
\hline Childcare / taking care of children & crying, cuddling, feeding, giggling, socializing \\
\hline Driving & driving, socializing \\
\hline Eating & chewing, eating \\
\hline Fighting & fighting \\
\hline Gardening & gardening, mowing, planting, shoveling, weeding \\
\hline Grooming & bathing, brushing, combing, trimming, washing \\
\hline Hiking & hiking \\
\hline Housework & cleaning, dusting, repairing, scrubbing, vacuuming \\
\hline Instructing & instructing, teaching \\
\hline Playing games & gambling, playing+fun, playing+videogames, socializing \\
\hline Preparing food & barbecuing, boiling, chopping, cooking, frying, grilling, rinsing, stirring \\
\hline Reading & reading \\
\hline Religious activities & praying, preaching \\
\hline Sleeping & resting, sleeping \\
\hline Socializing and social events & celebrating, dancing, marrying, singing, socializing, talking \\
\hline Sports & exercising, playing+sports, swimming, throwing \\
\hline Telephoning & calling, telephoning \\
\hline Working & working \\
\hline Control videos & blowing, floating, raining, shaking \\
\hline
\end{tabular}

Table 1. Activities from the ATUS included in each of the two stimulus sets, with the corresponding verb labels from the Moments in Time dataset. Note that control videos were only included in the first dataset. 
382 We curated an initial set of approximately 500 videos from the Moments in Time dataset (see

383 Supplementary Methods) by identifying the verb labels relevant to our chosen activities. We then

384 selected two subsets of videos (1) that sampled all activities in a balanced manner, and (2) where

385 sociality (as assessed through behavioral ratings, see below Section 2.3) was minimally

386 correlated to the number of agents (experimenter-labeled). For more details see Supplementary

387 Methods. These two features are difficult to disentangle in naturalistic stimulus sets, and we were

388 able to minimize, though not fully eliminate, this correlation (Figure 1a).

389 The first stimulus set contained 152 videos (8 videos per activity and 8 additional videos with no 390 agents) and was used in Experiment 1. The second stimulus set contained 65 videos (3-4 videos

391 per activity) and was used in Experiment 2. The videos were preprocessed to a framerate of 30

392 frames-per-second and resized to $600 \times 400$ pixels.

393 Behavior: Participants

\section{Behavioral ratings}

395 A total of 256 workers (202 after exclusions, located in the United States, worker age and gender

396 not recorded) from the online platform Amazon Mechanical Turk provided sociality, valence,

397 arousal, and activity ratings of the video stimuli, and 43 workers (35 after exclusions) provided

398 transitivity ratings.

\section{Multiple arrangement}

400 Two separate online multiple arrangement experiments were performed on each of the two 401 stimulus sets. A total of 374 workers from Amazon Mechanical Turk took part in Experiment 1

402 (300 after exclusions, located in the United States, worker age and gender not recorded).

403 Experiment 2 involved 58 participants (53 after exclusions, 31 female, 20 male, 1 non-binary, 1

404 not reported, mean age 19.38 \pm 1.09 ) recruited through the Department of Psychological and

405 Brain Sciences Research Portal at Johns Hopkins University.

406 All procedures for online data collection were approved by the Johns Hopkins University

407 Institutional Review Board and informed consent was obtained from all participants. 
Behavior: Experimental procedure

\section{Behavioral ratings}

410 Participants viewed subsets of 30-60 videos from the initially curated large-scale set and rated

411 the events depicted on a five-point scale. In a first set of experiments, the dimensions rated were:

412 sociality (how social the events were, from 1 - not at all to 5 - very social); valence (how

413 pleasant the events were, from 1 - very unpleasant to 5 - very pleasant); arousal (how intense the

414 events were, from 1 - very calm to 5 - very intense); and activity (how active they were, from 1

415 - no action to 5 - very active). In separate experiments, participants provided transitivity ratings

416 for the two final stimulus sets (i.e. to what extent the actions involved a person or people

417 interacting with an object, from 1 - not at all to 5 - very much). This amounted to an average of

418 17.46 \pm 2.14 ratings per video (Experiment 1) and 18.22 \pm 2.09 ratings per video (Experiment 2).

419 The experiments were implemented in JavaScript using the jsPsych library (de Leeuw, 2015).

\section{Multiple arrangement}

421 To characterize human action representations, we collected behavioral similarity ratings using

422 two multiple arrangement experiments. The experiments were conducted on the Meadows

423 platform (www.meadows-research.com) and required participants to arrange the videos

424 according to their similarity inside a circular arena. Participants were free to use their own

425 criteria to determine similarity, so as to encourage natural behavior.

426 Each trial started with the videos arranged around the circular arena. The videos would start

427 playing on hover, and the trial would not end until all videos were played and dragged-and-

428 dropped inside the arena (Figure 2). Different sets of videos were presented in different trials. An

429 adaptive "lift-the-weakest" algorithm was used to resample the video pairs placed closest

430 together, so as to gather sufficient evidence (or improve the signal-to-noise ratio) for each pair.

431 This procedure was repeated until an evidence criterion of 0.5 was reached for each pair, or until

432 the experiment timed out (Experiment 1:90 minutes, Experiment 2: 120 minutes). By asking

433 participants to zoom into the subsets previously judged as similar, the task required the use of

434 different contexts and criteria to judge relative similarities. Compared to other methods of

435 measuring similarity, multiple arrangement thus combines efficient sampling of a large stimulus 
436 set with adaptive behavior that can recover a multi-dimensional similarity structure

437 (Kriegeskorte and Mur, 2012).

438 In Experiment 1, participants arranged different subsets of 30 videos from the 152-video set, 439 with a maximum of 7 videos shown in any one trial. The stimuli were sampled in a balanced 440 manner across participants. The task took on average $32 \pm 14.4$ minutes and $86.8 \pm 22.6$ trials.

441 In Experiment 2, all participants arranged the same 65 videos (entire 65-video set), with a 442 maximum of 8 videos shown in any one trial. The task took on average $87.5 \pm 24.6$ minutes, 443 including breaks, and $289.7 \pm 57.3$ trials.

444 Both experiments included a training trial in which participants arranged the same 7 videos 445 before beginning the main task. Participants were excluded from further analysis if there was a 446 low correlation between their training data and the average of all other participants' data (over 2 447 standard deviations below the mean). They were also excluded if they responded incorrectly to a 448 catch trial requiring them to label the action in previously seen videos.

449 Inverse MDS was used to construct behavioral dissimilarity matrices containing normalized 450 Euclidean distances between all pairs of videos (Kriegeskorte and Mur, 2012). In Experiment 1, 451 the behavioral RDM contained 11476 pairs with an average of $11.37 \pm 3.08$ ratings per pair; in 452 Experiment 2, there were 2080 pairs rated by all 53 participants.

453 Behavior: Data analysis

454 Representational similarity analysis

455 Everyday actions can be differentiated along numerous axes. Perceptually, they can differ in 456 terms of visual properties, like the setting in which they take place. They can also be 457 characterized through action-related features like semantic action category, or through social 458 features, like the number of agents involved. Understanding how these features contribute to 459 natural behavior can shed light on how naturalistic action representations are organized. Here, 460 we used representational similarity analysis (RSA) to assess the contribution of visual, action, 461 and social-affective features to the behavioral similarity data. 
462 We quantified features of interest using image properties, labels assigned by experimenters, and

463 behavioral ratings (provided by participants, see above Section 2.3). We calculated the Euclidean

464 distances between all pairs of stimuli in each feature space, thus generating 17 feature RDMs.

465 To quantify visual features, image properties were extracted separately for each frame of each

466 video and averaged across frames. These included pixel value, hue, saturation, motion energy

467 (the magnitude of the optic flow estimated using the Horn-Schunck method), and the spatial

468 envelope of each image quantified using GIST (Oliva and Torralba, 2001). We also extracted

469 activations from the first convolutional layer and last fully-connected layer of a pre-trained

470 feedforward convolutional neural network (AlexNet; Krizhevsky et al., 2012). Two additional

471 experimenter-labeled features were included: scene setting (indoors/outdoors) and the presence

472 of a watermark.

473 Action feature RDMs were based on transitivity and activity ratings (provided by participants,

474 see above), as well as action category (a binary RDM clustering the stimuli into activity

475 categories based on the initial dataset designations) and effectors (experimenter-labeled). The

476 latter consisted of binary vectors indicating the involvement of body parts in each action

477 (face/head, hands, arms, legs, and torso). To assess whether a more detailed semantic model

478 would capture more information, we also performed a control analysis using a feature RDM

479 based on WordNet similarities between the verb labels in the "Moments in Time" dataset

480 (Supplementary Figure 2).

481 Social-affective feature RDMs were based on sociality, valence, and arousal ratings (all provided

482 by participants, see Behavioral Ratings above) and the number of agents in each video, which

483 was labeled by experimenters on a four-point scale (from 0, no agent present, to 3, three or more

484 agents present).

485 Each subject's behavioral RDM was correlated to the feature RDMs, and the resulting Kendall's

$486 \tau_{A}$ values were tested against chance using one-tailed sign permutation testing (5000 iterations).

487 P-values were omnibus-corrected for multiple comparisons using a maximum correlation

488 threshold across all models (Nichols and Holmes, 2001). 
489 A noise ceiling was calculated by correlating each subject's RDM to the average RDM (upper

490 bound), as well as to the average RDM excluding the left-out subject (lower bound; Nili et al.,

491 2014).

\section{Variance partitioning}

493 Despite low correlations between features of interest in both stimulus sets (Figure 1a), shared

494 variance could still contribute to the representational similarity analysis results. To estimate the

495 unique contributions of the three primary groups of features, we performed a cross-validated

496 variance partitioning analysis, excluding individual features that did not correlate with the

497 behavioral data in the above RSA analysis. The three groups included: visual features (scene

498 setting and the last fully-connected layer of AlexNet), action features (action category, effectors,

499 transitivity, action), and social-affective features (number of agents, sociality, valence, arousal).

500 The behavioral data were randomly split into training and test sets (100 iterations) by leaving out

501 half of the individual ratings for each pair of videos in Experiment 1 (since different participants

502 saw different subsets of videos) or half of the participants in Experiment 2. We fit seven different

503 regression models using the average training RDM (with every possible combination of the three

504 groups of features), and we calculated the squared Kendall's $\tau_{A}$ between the predicted responses

505 and the average test RDM. These values were then used to calculate the unique and shared

506 portions of variance contributed by the predictors (Groen et al., 2018; Lescroart et al., 2015;

507 Tarhan et al., 2021).

508 The resulting values were tested against chance using one-tailed sign permutation testing (5000

509 iterations, omnibus-corrected for multiple comparisons). Differences between groups of features

510 were assessed with two-sided Wilcoxon signed-rank tests.

\section{EEG: Stimuli}

512 The stimulus set from behavioral Experiment 1 was used in the EEG experiment, containing 152

513 videos from 18 categories, as well as control videos. The three-second stimuli were trimmed to a

514 duration of 0.5 seconds centered around the action to improve time-locking to the EEG signals

515 and allow for a condition-rich experimental design. An additional 50 videos were included as

516 catch stimuli (25 pairs depicting the same action, manually chosen from the larger stimulus set). 


\section{EEG: Participants}

518 Fifteen participants (6 female, 9 male, mean age $25.13 \pm 6.81$ ) took part in the EEG experiment.

519 All participants were right-handed and had normal or corrected-to-normal vision. Informed

520 consent was obtained in accordance with the Declaration of Helsinki and all procedures were

521 approved by the Johns Hopkins University Institutional Review Board.

\section{EEG: Experimental procedure}

523 Continuous EEG recordings with a sampling rate of $1000 \mathrm{~Hz}$ were made with a 64-channel Brain

524 Products ActiCHamp system using actiCAP electrode caps in a Faraday chamber. Electrode

525 impedances were kept below $25 \mathrm{k} \Omega$ when possible and the $\mathrm{Cz}$ electrode was used as an online

526 reference.

527 Participants were seated upright while viewing the videos on a back-projector screen situated

528 approximately $45 \mathrm{~cm}$ away. The 152 videos were shown in pseudorandom order in each of 10

529 blocks with no consecutive repetition allowed. In addition, four repetitions of the 25 catch video

530 pairs were presented at random times during the experiment, with the pairs shuffled to minimize

531 learning effects. Participants performed a one-back task and were asked to press a button on a

532 Logitech game controller when they detected two consecutive videos showing the same action.

533 There was a break every 150 trials and participants could continue the experiment by pressing a

534 button. In total, the experiment consisted of 1720 trials (1520 experimental trials and 200 catch

535 trials) and took approximately 45 minutes.

536 Each trial started with a black fixation cross presented on a gray screen for a duration chosen

537 from a uniform distribution between 1 and $1.5 \mathrm{~s}$, followed by a $0.5 \mathrm{~s}$ video. The stimuli were

538 presented on the same gray background and subtended approximately $15 \times 13$ degrees of visual

539 angle. The fixation cross remained on screen and participants were asked to fixate throughout the

540 experiment. A photodiode was used to accurately track on-screen stimulus presentation times

541 and account for projector lag. The paradigm was implemented in MATLAB R2019a using the

542 Psychophysics Toolbox (Brainard, 1997; Kleiner et al., 2007; Pelli, 1997). 


\section{EEG: Data analysis}

\section{Preprocessing}

545 EEG data preprocessing was performed using MATLAB R2020b and the FieldTrip toolbox

546 (Oostenveld et al., 2011). First, the EEG data were aligned to stimulus onset using the

547 photodiode data to correct for any lag between stimulus triggers and on-screen presentation. The

548 aligned data were segmented into $1.2 \mathrm{~s}$ epochs $(0.2 \mathrm{~s}$ pre-stimulus to $1 \mathrm{~s}$ post-stimulus onset),

549 baseline-corrected using the $0.2 \mathrm{~s}$ prior to stimulus onset, and high-pass filtered at $0.1 \mathrm{~Hz}$.

550 Artefact rejection was performed using a semi-automated pipeline. First, the data were filtered 551 between 110 and $140 \mathrm{~Hz}$ and Hilbert-transformed to detect muscle artefacts; segments with a z-

552 value cutoff above 15 were removed. Next, channels and trials with high variance were manually

553 rejected based on visual inspection of a summary plot generated using the $f t$ rejectvisual

554 function in FieldTrip. Finally, independent component analysis (ICA) was performed to identify

555 and remove eye movement components from the data.

556 Catch trials were removed from the data together with any trials that elicited a button response

$557(13.74 \% \pm 1.82 \%$ of all trials). Of the remaining trials, $8.36 \% \pm 5.01 \%$ (ranging between 25 and

558275 trials) were removed during the artefact rejection procedure. A maximum of two noisy

559 electrodes were removed from eight participants' datasets.

560 Prior to further analysis, the data were re-referenced to the median across all electrodes, low-pass

561 filtered at $30 \mathrm{~Hz}$ to investigate evoked responses and downsampled to $500 \mathrm{~Hz}$.

\section{Multivariate analysis}

563 We performed multivariate analyses to investigate (1) whether EEG patterns reflected behavioral 564 similarity, and (2) whether different visual, action, and social-affective features explained 565 variance in the neural data.

566 First, time-resolved decoding of every pair of videos was performed using a linear Support 567 Vector Machine classifier as implemented in the LibSVM library (Chang and Lin, 2011). For 568 each pair of videos, pseudotrials were created by splitting each participant's single-trial data into 
569 two subsets and averaging the trials in each subset to improve SNR (Isik et al., 2018). The two

570 pseudotrials were used to train and test the classifier separately at each timepoint, and

571 multivariate noise normalization was performed using the covariance matrix of the training data

572 (Guggenmos et al., 2018). This procedure was repeated 10 times with different data splits. The

573 average decoding accuracies between all pairs of videos were used to generate a time-resolved

574 neural RDM for each participant, with decoding accuracy being used as a measure of

575 dissimilarity.

576 Next, we evaluated the correlations between each participant's neural RDM and the feature

577 RDMs found to correlate with behavioral similarity (Experiment 1). To investigate the link

578 between behavioral and neural representations, we also correlated neural RDMs with the average

579 behavioral RDM obtained from the multiple arrangement task in Experiment 1. This analysis

580 was performed using $10 \mathrm{~ms}$ sliding windows with an overlap of $6 \mathrm{~ms}$. The resulting Kendall's $\tau_{A}$

581 values were tested against chance using one-tailed sign permutation testing (5000 iterations,

582 cluster-corrected for multiple comparisons across time using the maximum cluster sum, $\alpha=$

5830.05 , cluster-setting $\alpha=0.05)$. A noise ceiling was calculated using the same procedure as in the

584 behavioral RSA (see above Section 2.4). Effect latencies were assessed by bootstrapping the

585 individual correlations 1000 times with replacement to calculate $90 \%$ confidence intervals

586 around effect onsets.

587 To quantify the contributions of visual, social-affective, and action features to the neural RDMs,

588 a time-resolved cross-validated variance partitioning procedure was performed. Using 100 split-

589 half cross-validation iterations, the neural RDM was entered as a response variable in a

590 hierarchical regression with three groups of feature RDMs (visual, social-affective, and action)

591 as predictors. This analysis employed the same ten feature RDMs used in the behavioral variance

592 partitioning (see above Variance partitioning), with the addition of activations from the first

593 convolutional layer of AlexNet (Conv1). As Conv1 best captures early visual responses

594 (Supplementary Figure 7), its inclusion ensured that we did not underestimate the role of visual

595 features in explaining neural variance.

596 The analysis was carried out using $10 \mathrm{~ms}$ sliding windows with an overlap of $6 \mathrm{~ms}$. The resulting

597 predicted Kendall's $\tau_{A}$ values were tested against chance using one-tailed sign permutation 
598 testing (5000 iterations, cluster-corrected for multiple comparisons using the maximum cluster

599 sum across time windows and regressions performed, $\alpha=0.05$, cluster-setting $\alpha=0.05$ ). The

600 distributions of effect onsets across the 100 split-half iterations were compared using two-sided

601 Wilcoxon signed-rank tests.

602

\section{Data availability}

603 Behavioral and EEG data and results have been archived as an Open Science Framework

604 repository (https://osf.io/hrmxn/). Analysis code is available on GitHub

605 (https://github.com/dianadima/mot_action).

606 Acknowledgments

607 This material is based upon work supported by the Center for Brains, Minds and Machines

608 (CBMM), funded by NSF STC award CCF-1231216. The authors wish to thank Tara Ghazi,

609 Seah Chang, Alyssandra Valenzuela, Melody Lee, Cora Mentor Roy, Haemy Lee Masson, and

610 Lucy Chang for their help with the EEG data collection, Dimitrios Pantazis for pairwise

611 decoding code, and Emalie McMahon for comments on the manuscript.

\section{References}

614 Allen, M., Poggiali, D., Whitaker, K., Marshall, T.R., Kievit, R.A., 2019. Raincloud plots: A

615 multi-platform tool for robust data visualization. Wellcome Open Res. 4.

616 https://doi.org/10.12688/wellcomeopenres.15191.1

617 ATUS, 2019. American Time Use Survey, United States Department of Labor. Bureau of Labor 618 Statistics. Washington, DC.

619 Bar, M., 2004. Visual objects in context. Nat. Rev. Neurosci. 5, 617-629.

620 https://doi.org/10.1038/nrn1476

621 Bedny, M., Caramazza, A., 2011. Perception, action, and word meanings in the human brain:

622 The case from action verbs. Ann. N. Y. Acad. Sci. https://doi.org/10.1111/j.1749- 


$$
6632.2011 .06013 . x
$$

624 Bellot, E., Abassi, E., Papeo, L., 2021. Moving Toward versus Away from Another: How Body Motion Direction Changes the Representation of Bodies and Actions in the Visual Cortex.

Brainard, D.H., 1997. The Psychophysics Toolbox. Spat. Vis. 10, 433-436. Cereb. Cortex 1-16. https://doi.org/10.1093/cercor/bhaa382

Carlson, T., Tovar, D.A., Alink, A., Kriegeskorte, N., 2013. Representational dynamics of object

Chang, C.C., Lin, C.J., 2011. LIBSVM: A Library for support vector machines. ACM Trans. Intell. Syst. Technol. 2. https://doi.org/10.1145/1961189.1961199

Cichy, R.M., Pantazis, D., Oliva, A., 2014. Resolving human object recognition in space and

de Leeuw, J.R., 2015. jsPsych: A JavaScript library for creating behavioral experiments in a Web browser. Behav. Res. Methods 47, 1-12. https://doi.org/10.3758/s13428-014-0458-y

Dijkstra, N., Ambrogioni, L., Vidaurre, D., van Gerven, M., 2020. Neural dynamics of perceptual inference and its reversal during imagery. Elife 9, 1-19.

Dima, D.C., Perry, G., Messaritaki, E., Zhang, J., Singh, K.D., 2018. Spatiotemporal dynamics in human visual cortex rapidly encode the emotional content of faces. Hum. Brain Mapp. 39,

Giese, M.A., Poggio, T., 2003. Cognitive neuroscience: Neural mechanisms for the recognition 3993-4006. https://doi.org/10.1002/hbm.24226

645 Greene, M.R., Hansen, B.C., 2018. Shared spatiotemporal category representations in biological and artificial deep neural networks. PLOS Comput. Biol. 14, e1006327. 
Groen, I.I., Greene, M.R., Baldassano, C., Fei-Fei, L., Beck, D.M., Baker, C.I., 2018. Distinct contributions of functional and deep neural network features to representational similarity of scenes in human brain and behavior. Elife 7, e32962. https://doi.org/10.7554/eLife.32962

Guggenmos, M., Sterzer, P., Cichy, R.M., 2018. Multivariate pattern analysis for MEG: A comparison of dissimilarity measures. Neuroimage 173, 434-447.

Hafri, A., Trueswell, J.C., Epstein, R.A., 2017. Neural representations of observed actions generalize across static and dynamic visual input. J. Neurosci. 37, 3056-3071.

Haxby, J. V., Gobbini, M.I., Nastase, S.A., 2020. Naturalistic stimuli reveal a dominant role for agentic action in visual representation. Neuroimage 216, 116561.

Hirai, M., Fukushima, H., Hiraki, K., 2003. An event-related potentials study of biological motion perception in humans. Neurosci. Lett. 344, 41-44. https://doi.org/10.1016/S0304-

Hirai, M., Hiraki, K., 2006. The relative importance of spatial versus temporal structure in the perception of biological motion: An event-related potential study. Cognition 99, 15-29. https://doi.org/10.1016/j.cognition.2005.05.003

Hochstein, S., Ahissar, M., 2002. View from the Top: Hierarchies and Reverse Hierarchies in the Visual System. Neuron 36, 791-804.

Humphreys, G.F., Newling, K., Jennings, C., Gennari, S.P., 2013. Motion and actions in language: Semantic representations in occipito-temporal cortex. Brain Lang. 125, 94-105. https://doi.org/10.1016/j.bandl.2013.01.008 posterior superior temporal sulcus. Proc. Natl. Acad. Sci. U. S. A. 114, E9145-E9152. https://doi.org/10.1073/pnas.1714471114 
674 Isik, L., Meyers, E.M., Leibo, J.Z., Poggio, T., 2014. The dynamics of invariant object

675

676 recognition in the human visual system. J. Neurophysiol. 111, 91-102. https://doi.org/10.1152/jn.00394.2013

Isik, L., Tacchetti, A., Poggio, T., 2018. A fast, invariant representation for human action in the visual system. J. Neurophysiol. 119, 631-640. https://doi.org/10.1152/jn.00642.2017

Jamali, M., Grannan, B.L., Fedorenko, E., Saxe, R., Báez-Mendoza, R., Williams, Z.M., 2021. Single-neuronal predictions of others' beliefs in humans. Nature 591, 610-614. https://doi.org/10.1038/s41586-021-03184-0

Johansson, G., 1973. Visual perception of biological motion and a model for its analysis. Percept. Psychophys. 14, 201-211. https://doi.org/10.3758/BF03212378

Jokisch, D., Daum, I., Suchan, B., Troje, N.F., 2005. Structural encoding and recognition of biological motion: Evidence from event-related potentials and source analysis. Behav. Brain Res. 157, 195-204. https://doi.org/10.1016/j.bbr.2004.06.025

Kleiner, M., Brainard, D.H., Pelli, D.G., Broussard, C., Wolf, T., Niehorster, D., 2007. What's new in Psychtoolbox-3? Perception 36, S14. https://doi.org/10.1068/v070821

Kriegeskorte, N., Mur, M., 2012. Inverse MDS: Inferring dissimilarity structure from multiple item arrangements. Front. Psychol. 3, 1-13. https://doi.org/10.3389/fpsyg.2012.00245

Krizhevsky, A., Sutskever, I., Hinton, G.E., 2012. ImageNet Classification with Deep Convolutional Neural Networks. Adv. Neural Inf. Process. Syst. 1-9. https://doi.org/http://dx.doi.org/10.1016/j.protcy.2014.09.007

Lescroart, M.D., Stansbury, D.E., Gallant, J.L., 2015. Fourier power, subjective distance, and object categories all provide plausible models of BOLD responses in scene-selective visual areas. Front. Comput. Neurosci. 9, 1-20. https://doi.org/10.3389/fncom.2015.00135 Sci. 19, 268-277. https://doi.org/10.1016/j.tics.2015.03.006 
Monfort, M., Andonian, A., Zhou, B., Ramakrishnan, K., Bargal, S.A., Yan, T., Brown, L., Fan, Q., Gutfruend, D., Vondrick, C., Oliva, A., 2019. Moments in Time Dataset: one million videos for event understanding. IEEE Trans. Pattern Anal. Mach. Intell. 1-8.

Nastase, S.A., Goldstein, A., Hasson, U., 2020. Keep it real: rethinking the primacy of experimental control in cognitive neuroscience. Neuroimage 222, 117254. https://doi.org/10.1016/j.neuroimage.2020.117254

Nichols, T.E., Holmes, A.P., 2001. Nonparametric Permutation Tests For Functional Neuroimaging : A Primer with Examples. Hum. Brain Mapp. 25, 1-25. https://doi.org/10.1002/hbm.1058

Nili, H., Wingfield, C., Walther, A., Su, L., Marslen-Wilson, W., Kriegeskorte, N., 2014. A Toolbox for Representational Similarity Analysis. PLoS Comput. Biol. 10, e1003553. https://doi.org/10.1371/journal.pcbi.1003553

Oliva, A., Torralba, A., 2001. Modeling the Shape of the Scene : A Holistic Representation of the Spatial Envelope. Int. J. Comput. Vis. 42, 145-175. https://doi.org/10.1023/A:1011139631724

Oostenveld, R., Fries, P., Maris, E., Schoffelen, J.M., 2011. FieldTrip: Open source software for advanced analysis of MEG, EEG, and invasive electrophysiological data. Comput. Intell. Neurosci. 2011, 156869. https://doi.org/10.1155/2011/156869

Papeo, L., 2020. Twos in human visual perception. Cortex 132, 473-478. https://doi.org/10.1016/j.cortex.2020.06.005

Pelli, D.G., 1997. The VideoToolbox software for visual psychophysics: Transforming numbers into movies. Spat. Vis. 10, 437-442. https://doi.org/10.1163/156856897X00366

Pitcher, D., Ungerleider, L.G., 2021. Evidence for a Third Visual Pathway Specialized for Social Perception. Trends Cogn. Sci. https://doi.org/10.1016/j.tics.2020.11.006

Quadflieg, S., Koldewyn, K., 2017. The neuroscience of people watching: How the human brain makes sense of other people's encounters. Ann. N. Y. Acad. Sci. 1396, 166-182. 
https://doi.org/10.1111/nyas.13331

726

727

Redcay, E., Moraczewski, D., 2020. Social cognition in context: A naturalistic imaging approach. Neuroimage 216, 116392. https://doi.org/10.1016/j.neuroimage.2019.116392

Spunt, R.P., Satpute, A.B., Lieberman, M.D., 2011. Identifying the what, why, and how of an observed action: An fMRI study of mentalizing and mechanizing during action observation. J. Cogn. Neurosci. 23, 63-74. https://doi.org/10.1162/jocn.2010.21446

Tarhan, L., De Freitas, J., Konkle, T., 2021. Behavioral and Neural Representations en route to Intuitive Action Understanding 1-22.

Tarhan, L., Konkle, T., 2020. Sociality and interaction envelope organize visual action representations. Nat. Commun. 11, 1-11. https://doi.org/10.1038/s41467-020-16846-w

Thornton, M.A., Tamir, D.I., 2021. People accurately predict the transition probabilities between actions. Sci. Adv. 7, eabd4995. https://doi.org/10.1126/sciadv.abd4995

Thornton, M.A., Weaverdyck, M.E., Tamir, D.I., 2019. The brain represents people as the mental states they habitually experience. Nat. Commun. 10, 1-10. https://doi.org/10.1038/s41467019-10309-7

Tucciarelli, R., Turella, L., Oosterhof, N.N., Weisz, N., Lingnau, A., 2015. MEG Multivariate Analysis Reveals Early Abstract Action Representations in the Lateral Occipitotemporal Cortex. J. Neurosci. 35, 16034-16045. https://doi.org/10.1523/JNEUROSCI.1422-15.2015

Tucciarelli, R., Wurm, M., Baccolo, E., Lingnau, A., 2019. The representational space of observed actions. Elife 8, 1-24. https://doi.org/10.7554/eLife.47686

Vangeneugden, J., Peelen, M. V., Tadin, D., Battelli, L., 2014. Distinct neural mechanisms for body form and body motion discriminations. J. Neurosci. 34, 574-585. https://doi.org/10.1523/JNEUROSCI.4032-13.2014

Wamain, Y., Pluciennicka, E., Kalénine, S., 2014. Temporal dynamics of action perception: Differences on ERP evoked by object-related and non-object-related actions. 
Neuropsychologia 63, 249-258. https://doi.org/10.1016/j.neuropsychologia.2014.08.034

751 Weaverdyck, M.E., Thornton, M.A., Tamir, D.I., 2021. The representational structure of mental states generalizes across target people and stimulus modalities. Neuroimage 238, 118258. https://doi.org/10.1016/j.neuroimage.2021.118258

Willems, R.M., Peelen, M. V., 2021. How context changes the neural basis of perception and language. iScience 24, 102392. https://doi.org/10.1016/j.isci.2021.102392

756 Wurm, M.F., Caramazza, A., 2019. Lateral occipitotemporal cortex encodes perceptual components of social actions rather than abstract representations of sociality. Neuroimage 202. https://doi.org/10.1016/j.neuroimage.2019.116153

Wurm, M.F., Caramazza, A., Lingnau, A., 2017. Action categories in lateral occipitotemporal cortex are organized along sociality and transitivity. J. Neurosci. 37, 562-575. https://doi.org/10.1523/JNEUROSCI.1717-16.2016

Wurm, M.F., Cramon, D.Y., Schubotz, R.I., 2012. The Context-Object-Manipulation triad: Cross Talk during action perception revealed by fMRI. J. Cogn. Neurosci. 24, 1548-1559. https://doi.org/10.1162/jocn_a_00232

765 Wurm, X.M.F., Lingnau, A., 2015. Decoding Actions at Different Levels of Abstraction. J. 766 Neurosci. 35, 7727-7735. https://doi.org/10.1523/JNEUROSCI.0188-15. 